\title{
Evolving outcomes of extracorporeal membrane oxygenation support for severe COVID-19 ARDS in Sorbonne hospitals, Paris
}

\author{
Matthieu Schmidt ${ }^{1,2,3^{*}+}$, Elise Langouet ${ }^{2 \dagger}{ }^{\text {, David Hajage }}{ }^{4}$, Sarah Aissi James ${ }^{2}$, Juliette Chommeloux ${ }^{1,2}$, \\ Nicolas Bréchot ${ }^{1,2}$, Petra Barhoum 1,2, Lucie Lefèvre ${ }^{1,2}$, Antoine Troger ${ }^{1,2}$, Marc Pineton de Chambrun ${ }^{1,2}$, \\ Guillaume Hékimian 1,2, Charles-Edouard Luyt ${ }^{1,2}$, Martin Dres 3,5,6, Jean-Michel Constantin ${ }^{7}$, Muriel Fartoukh ${ }^{3,8}$, \\ Pascal Leprince ${ }^{1,9}$, Guillaume Lebreton ${ }^{1,9}$, Alain Combes ${ }^{1,2,3}$ and for the GRC RESPIRE Sorbonne Université
}

\begin{abstract}
Background: Extracorporeal membrane oxygenation (ECMO) was frequently used to treat patients with severe coronavirus disease-2019 (COVID-19)-associated acute respiratory distress (ARDS) during the initial outbreak. Care of COVID-19 patients evolved markedly during the second part of 2020. Our objective was to compare the characteristics and outcomes of patients who received ECMO for severe COVID-19 ARDS before or after July 1, 2020.

Methods: We included consecutive adults diagnosed with COVID-19 in Paris-Sorbonne University Hospital Network ICUs, who received ECMO for severe ARDS until January 28, 2021. Characteristics and survival probabilities over time were estimated during the first and second waves. Pre-ECMO risk factors predicting 90-day mortality were assessed using multivariate Cox regression.

Results: Characteristics of the 88 and 71 patients admitted, respectively, before and after July 1, 2020, were comparable except for older age, more frequent use of dexamethasone (18\% vs. 82\%), high-flow nasal oxygenation (19\% vs. $82 \%$ ) and/or non-invasive ventilation (7\% vs. 37\%) after July 1. Respective estimated probabilities (95\% confidence intervals) of 90-day mortality were $36 \%$ (27-47\%) and $48 \%$ (37-60\%) during the first and the second periods. After adjusting for confounders, probability of 90-day mortality was significantly higher for patients treated after July 1 (HR 2.27, 95\% Cl 1.02-5.07). ECMO-related complications did not differ between study periods.
\end{abstract}

Conclusions: 90 -day mortality of ECMO-supported COVID-19-ARDS patients increased significantly after July 1 , 2020, and was no longer comparable to that of non-COVID ECMO-treated patients. Failure of prolonged non-invasive oxygenation strategies before intubation and increased lung damage may partly explain this outcome.

Keywords: Extracorporeal membrane oxygenation, Venovenous ECMO, Acute respiratory distress syndrome (ARDS), COVID-19, SARS-CoV-2, Outcomes

\footnotetext{
*Correspondence: matthieu.schmidt@aphp.fr

${ }^{\dagger}$ Matthieu Schmidt and Elise Langouet jointly first authors

${ }^{2}$ Service de Médecine Intensive-Réanimation, iCAN, Institut de Cardiologie, Assistance Publique-Hôpitaux de Paris (APHP), Sorbonne Université Hôpital Pitié-Salpêtrière, 47, Bd de L'Hôpital, 75651 Paris Cedex 13, France

Full list of author information is available at the end of the article
}

\begin{abstract}
Introduction
Extracorporeal membrane oxygenation (ECMO) was frequently used to treat coronavirus disease-2019 (COVID19) patients with severe acute respiratory distress syndrome (ARDS) during the initial outbreak from January to June 2020 [1-4]. High-volume ECMO centers and large ECMO networks reported similar survival rates for
\end{abstract}

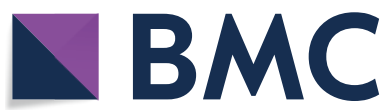

(c) The Author(s) 2021. Open Access This article is licensed under a Creative Commons Attribution 4.0 International License, which permits use, sharing, adaptation, distribution and reproduction in any medium or format, as long as you give appropriate credit to the original author(s) and the source, provide a link to the Creative Commons licence, and indicate if changes were made. The images or other third party material in this article are included in the article's Creative Commons licence, unless indicated otherwise in a credit line to the material. If material is not included in the article's Creative Commons licence and your intended use is not permitted by statutory regulation or exceeds the permitted use, you will need to obtain permission directly from the copyright holder. To view a copy of this licence, visit http://creativecommons.org/licenses/by/4.0/. The Creative Commons Public Domain Dedication waiver (http://creativeco mmons.org/publicdomain/zero/1.0/) applies to the data made available in this article, unless otherwise stated in a credit line to the data. 
these patients compared to ECMO-supported patients with non-COVID-associated ARDS [5-7].

Management of COVID-19 patients evolved over the following months, as knowledge of the disease improved with the publication of landmark randomized trials. In June 2020, RECOVERY-trial results showed that dexamethasone (6 mg/day for 10 days) significantly reduced mortality compared to usual care for patients receiving either invasive mechanical ventilation or oxygen alone. That dexamethasone regimen was recommended by the World Health Organization and largely prescribed to COVID-19 patients. High-flow oxygen through a nasal cannula (HFNO) or noninvasive ventilation (NIV) was also shown to lower COVID-19 patients' need for invasive mechanical ventilation and was recommended in national and international guidelines.

Because the care of COVID-19 patients evolved during the second part of 2020, we compared the characteristics and outcomes of patients who received ECMO support before and after July 1, 2020, for laboratory-confirmed severe acute respiratory distress syndrome (ARDS)-associated coronavirus-2 (SARS-CoV-2) infection in ParisSorbonne Hospitals.

\section{Methods}

\section{Study settings}

All consecutive adults, with laboratory-confirmed SARSCov-2 infection admitted to Paris-Sorbonne University Hospital Network ICUs, who received venoarterial (VA) - or venovenous (VV)-ECMO for severe ARDS from March 8, 2020, to January 28, 2021, were included, and provided 90-day survival status. The characteristics and outcomes of the first 83 cohort patients were reported previously [2]. We chose to split that cohort into two periods, before and after July 1,2020 , respectively. That date corresponds to the publication of the RECOVERY trial [8], and the transition period between the first and second COVID-19 waves in France. ECMO support was provided in four ICUs at Pitié-Salpêtrière and Tenon Hospitals before July 1, and in one Pitié-Salpetrière Hospital ICU thereafter. The Sorbonne University Ethics Committee approved the study protocol (CER-SU-2020-46).

\section{ECMO indications and organization}

ECMO indications for COVID-19 patients are detailed elsewhere $[2,4]$ and did not change throughout the study period. Briefly, indication(s) for ECMO implantation were centralized and evaluated in staff meetings, including at least two intensivists. Severe ARDS patients eligible for ECMO had to fulfill EOLIA trial respiratory severity criteria [5], with pre-ECMO use of neuromuscular-blocking agents and prone-positioning strongly recommended. Similarly, ECMO contraindications were still age $>70$ years, severe comorbidities, cardiac arrest, refractory multiorgan failure or Simplified Acute Physiology Score (SAPS) II [9]>90, irreversible neurological injury and/or invasive mechanical ventilation for $>10$ days. Once the indication was retained, the PitiéSalpêtrière Mobile ECMO Retrieval Team, comprising a cardiovascular surgeon and a perfusionist, was sent at the patient's bedside for ECMO cannulation and retrieval to our department, as described previously [10,11].

\section{Management of ECMO for COVID-19}

Throughout the study period, femoral-jugular percutaneous cannulation under ultrasonography guidance with a large drainage femoral cannula (25-29Fr) was strongly recommended for VV-ECMO. Pump speed was adjusted to obtain $>90 \%$ arterial oxygen saturation. Optimal cannula positioning was verified by ultrasonography and chest X-ray. Because of frequent thromboembolic events on-ECMO, including massive pulmonary embolism [12], and based on our early experience [2], the targeted activated partial thromboplastin time (aPTT) for VV-ECMO anticoagulation with unfractionated heparin was $60-75 \mathrm{~s}$ or anti-Xa activity $0.3-0.5 \mathrm{IU} / \mathrm{mL}$. The hemoglobin threshold for red-cell transfusion was 7-8 g/ $\mathrm{dL}$ and platelet transfusions were discouraged except for severe thrombocytopenia ( $<50 \mathrm{G} / \mathrm{L}$ ) or thrombocytopenia $<100 \mathrm{G} / \mathrm{L}$ with bleeding. To enhance protection against ventilator-induced lung injury, ultra-protective lung ventilation on-ECMO was recommended [6], and early prone-positioning on-ECMO was encouraged in the absence of hemodynamic instability and contraindications [13]. Based on the first descriptions [1, 2, 14] of very long ECMO runs and mechanical ventilation of COVID-19 patients, our team decided to decrease onECMO neuromuscular blocking-agent use for these patients. Patients were assessed daily for possible ECMOweaning using the EOLIA clinical and physiological criteria $[5,15]$. On-ECMO tracheostomy was considered after having identified, before the procedure, that the patient would likely tolerate decreased sedation. All tracheostomy decisions were made after discussion within the medical team of the balance between risks and benefits of that procedure on-ECMO [16]. When VA-ECMO was initiated, the ipsilateral limb received percutaneous cannulation via an anterograde perfusion catheter.

\section{Data collection}

Our first-wave, ECMO-treated COVID-19 patients' data were reported previously [2]. Briefly, they included pre-ECMO demographic information; severity scores (i.e., SAPS II [9]; Sequential Organ-Failure Assessment (SOFA) score [17]; Respiratory Extracorporeal 
Membrane Oxygenation Survival Prediction (RESP) score [18]); rescue therapies; pre-ECMO ventilatory mechanics, including driving pressure $(\Delta \mathrm{P})$, mechanical power [19] and ventilatory ratio [20]; arterial blood-gas parameters, and routine laboratory values. Because we expected that pre-intubation management had changed over the study period, HFNO and NIV use and their durations were also noted.

Mechanical ventilation settings, arterial blood gases, adjuvant therapies on-ECMO and ECMO-related complications were recorded daily from day 1 to 7 , then every 7 days until ECMO-day 60, ECMO-weaning or death, whichever occurred first. Major bleeding was defined as the need for $\geq 2$ units of packed red blood cells for an obvious hemorrhagic event, necessitating a surgical or interventional procedure, an intracerebral hemorrhage or being fatal, while massive hemolysis was defined as plasma-free hemoglobin $>500 \mathrm{mg} / \mathrm{L}$ associated with clinical signs of hemolysis.

Lastly, specific COVID-19 treatments were recorded. We specified whether dexamethasone $(6 \mathrm{mg} /$ day $)$ was initiated before or during ECMO, and if the patient received high-dose corticosteroids, defined as $>1 \mathrm{mg} / \mathrm{kg} /$ day of prednisone or equivalent since ARDS onset, during the ECMO run [21].

\section{Outcomes}

Patient outcomes on days $28,40,50,60,70,80$ or 90 postECMO implantation included the following endpoints: on-ECMO, in-ICU and weaned-off ECMO, alive and out of ICU or died. The time spent in each of those four states until the specified days were also calculated. Other outcomes included ICU- and ECMO-related complications.

\section{Statistical analyses}

Patient characteristics are expressed as $n$ (\%) for categorical variables or median (interquartile range, IQR) for continuous parameters, as appropriate.

To describe patients' in-ICU trajectories over time, a multi-state model was used as in our previous study [2, 22]. Briefly, this framework considers that a patient can go through different states during follow-up. Herein, the starting time was the ECMO-initiation day, making onECMO the initial state for all patients, potentially followed by two intermediate states: in-ICU \& weaned-off ECMO or alive \& out of the ICU. Because patients could die at any time during follow-up, either in-ICU or after discharge, the death is the only final absorbing state (the final state that a patient can enter but once entered cannot be left). In this four-state model (Additional file 1), each box represents a state and each arrow represents possible transitions from one state to another.
After assessing patient status, participants who did not reach the final absorbing state were right-censored. A Cox model stratified on each possible transition state was fitted to estimate transition (from one state to another) and state-occupation (for each of the four states) probabilities over time; the percentages of patients occupying each possible state were represented simultaneously over time with a stacked probability plot and reported with their $95 \%$ confidence interval (CI) on days $28,40,50$, $60,70,80$ and 90 post-ECMO initiation. Another figure (Additional file 2 ) individually displays all possible transition probabilities from one state to another over time. Mean state-occupation times (i.e., the expected length of stay in each possible state of the multi-state model) were also reported at the same times. Finally, median onECMO duration and length of ICU stay were established. These analyses were computed separately for the first and second waves.

Pre-ECMO risk factors for 90-day mortality were assessed for the entire cohort using univariate and multivariate Cox regression models. The variables included in the multivariate model were defined a priori, without any variable selection. Multiple imputations were used to replace missing values when appropriate. Briefly, 10 copies of the dataset were created with the missing values replaced by imputed values, based on observed data including participants' outcomes and pre-ECMO characteristics. Each dataset was then analyzed and the results from each dataset were pooled into a final result applying Rubin's rule [23]. Hazard ratios and their 95\% CIs were estimated.

Finally, adjusted Kaplan-Meier probabilities of survival were estimated from the multivariate Cox regression model. Each subject's survival probability over time was estimated from the model, first considering all subjects included during the first wave, and second considering all subjects included during the second wave. Then, the survival probabilities were averaged across all individuals. Finally, unadjusted and adjusted Kaplan-Meier curves were plotted on the same figure.

All the analyses were computed at a two-sided $\alpha$ level of $5 \%$ with $\mathrm{R}$ software, version 4.0.3.

\section{Results}

\section{Pre-ECMO patient characteristics}

Eighty-eight patients were admitted before July 1, 2020, and 71 thereafter (Table 1). Briefly, patients admitted after July 1 were significantly older, while the numbers and types of failing organs at ECMO cannulation were comparable. The first COVID-19-symptoms-to-intubation interval was longer during the second period. Respective before and after HFNO (19\% vs. $82 \%)$ and/ or NIV (7\% vs. $37 \%)$ use and their durations before 
Table 1 Pre-ECMO characteristics according to ICU admission before or after July 1, 2020

\begin{tabular}{|c|c|c|c|c|}
\hline \multirow[t]{2}{*}{ Characteristic } & \multirow[t]{2}{*}{ All $(N=159)$} & \multicolumn{2}{|l|}{ ICU admission } & \multirow[t]{2}{*}{$p$} \\
\hline & & Before July $1(N=88)$ & After July $1(N=71)$ & \\
\hline Age, years & $51(43-58)$ & $49(41-56)$ & $54(49-60)$ & 0.002 \\
\hline Male & $114(72)$ & $64(73)$ & $50(70)$ & 0.784 \\
\hline Body mass index, $\mathrm{kg} / \mathrm{cm}^{2}$ & $30.8(27.7-35.1)$ & $30.3(27.7-34.0)$ & $31.0(27.2-37.0)$ & 0.513 \\
\hline SAPS $\|$ & 55 (39-64) & $46(30-57)$ & $61(53-68)$ & $<0.001$ \\
\hline RESP score & $4(2-5)$ & $4(2-5)$ & $3(2-4)$ & 0.020 \\
\hline Total SOFA score & $11(9-13)$ & $11(9-13)$ & $11(8-13)$ & 0.599 \\
\hline Renal component $\geq 3$ & $24(15)$ & $15(17)$ & $9(13)$ & 0.795 \\
\hline Cardiovascular component $\geq 3$ & $79(50)$ & $44(50)$ & $35(49)$ & 0.752 \\
\hline Hematological component $\geq 3$ & $4(3)$ & $2(2)$ & $2(3)$ & 0.556 \\
\hline \multicolumn{5}{|l|}{ Comorbidities } \\
\hline Hypertension & $64(40)$ & $34(39)$ & $30(42)$ & 0.644 \\
\hline Diabetes & $54(34)$ & $38(43)$ & $26(37)$ & 0.525 \\
\hline Chronic respiratory disease $\mathrm{a}^{\mathrm{a}}$ & $24(15)$ & $10(11)$ & $14(20)$ & 0.143 \\
\hline Immunocompromised $^{\mathrm{b}}$ & $9(6)$ & $3(3)$ & $6(8)$ & 0.189 \\
\hline \multicolumn{5}{|l|}{ Time from } \\
\hline First symptoms to ICU admission, days & $7(5-10)$ & $7(5-10)$ & $8(5-11)$ & 0.452 \\
\hline First symptoms to intubation, days & $10(7-13)$ & $8(6-11)$ & $11(8-17)$ & $<0.001$ \\
\hline ICU admission to intubation, days & $1(0-3)$ & $0(0-1)$ & $3(1-8)$ & $<0.001$ \\
\hline ICU admission to ECMO, days & $7(3-9)$ & $6(3-7)$ & $9(4-12)$ & $<0.001$ \\
\hline Intubation to ECMO, days & $4(1-7)$ & $4(3-6)$ & $3(1-7)$ & 0.115 \\
\hline MERT retrieval on-ECMO from another hospital & $135(85)$ & $66(75)$ & $69(97)$ & $<0.001$ \\
\hline High-flow oxygen before intubation & $75(47)$ & $17(19)$ & $58(82)$ & $<0.001$ \\
\hline Duration, days & $4(1-7)$ & $2(0-4)$ & $5(1-8)$ & 0.009 \\
\hline Non-invasive ventilation before intubation & $32(20)$ & $6(7)$ & $26(37)$ & $<0.001$ \\
\hline Duration, days & $3(1-6)$ & $2(3-4)$ & $3(1-8)$ & 0.575 \\
\hline Volume-assist control ventilation & $156(98)$ & $88(100)$ & $68(96)$ & 0.094 \\
\hline \multicolumn{5}{|l|}{ Ventilation parameters } \\
\hline $\mathrm{FiO}_{2}$ & $100(100-100)$ & $100(100-100)$ & $100(100-100)$ & 0.359 \\
\hline PEEP, $\mathrm{cmH}_{2} \mathrm{O}$ & $12(10-14)$ & $13(12-14)$ & $12(10-14)$ & 0.019 \\
\hline Tidal volume, mL/kg PBW & $6.0(5.6-6.4)$ & $6.0(5.7-6.4)$ & $6.1(5.5-6.3)$ & 0.661 \\
\hline Respiratory rate, breaths/min & $30(26-30)$ & $30(28-30)$ & $30(26-30)$ & 0.519 \\
\hline Plateau pressure, $\mathrm{CmH}_{2} \mathrm{O}$ & $31(29-32)$ & $31(29-33)$ & $30(30-32)$ & 0.717 \\
\hline Driving pressure, $\mathrm{CmH}_{2} \mathrm{O}^{\mathrm{c}}$ & $19(16-21)$ & $18(16-20)$ & $19(16-22)$ & 0.289 \\
\hline Static compliance, $\mathrm{mL} / \mathrm{cmH}_{2} \mathrm{O}$ & $21.5(17.5-26.4)$ & $22.1(18.1-26.5)$ & $20.8(17.0-23.9)$ & 0.148 \\
\hline Mechanical power, J/min ${ }^{d}$ & $23.8(20.2-27.1)$ & $24.9(21.9-27.2)$ & $21.4(19.2-26.4)$ & 0.055 \\
\hline Ventilatory ratio & $2.7(2.2-3.1)$ & $2.8(2.3-3.3)$ & $2.5(2.0-2.9)$ & 0.046 \\
\hline \multicolumn{5}{|l|}{ Last blood-gas values pre-ECMO } \\
\hline $\mathrm{pH}$ & $7.32(7.25-7.39)$ & $7.32(7.24-7.38)$ & $7.33(7.26-7.41)$ & 0.143 \\
\hline $\mathrm{PaO}_{2} / \mathrm{FiO}_{2}$ & $60(54-69)$ & $60(54-68)$ & $60(54-74)$ & 0.601 \\
\hline $\mathrm{PaCO}_{2}, \mathrm{mmHg}$ & $56(49-65)$ & $57(50-67)$ & $53(48-63)$ & 0.156 \\
\hline $\mathrm{PaO}_{2}, \mathrm{mmHg}$ & $63(54-70)$ & $64(54-71)$ & $63(55-70)$ & 0.857 \\
\hline Plasma bicarbonate, $\mathrm{mmol} / \mathrm{L}$ & $27(23-32)$ & $27(24-32)$ & $27(23-33)$ & 0.705 \\
\hline Arterial lactate, $\mathrm{mmol} / \mathrm{L}$ & $1.6(1.3-2.1)$ & $1.6(1.4-2.0)$ & $1.6(1.3-2.4)$ & 0.858 \\
\hline \multicolumn{5}{|l|}{ Laboratory values } \\
\hline White-cell count, G/L & $13.0(9.5-18.0)$ & $13.1(10.0-17.1)$ & $12.9(8.6-18.9)$ & 0.872 \\
\hline Lymphocytes, G/L & $0.85(0.50-1.33)$ & $0.96(0.55-1.46)$ & $0.80(0.50-1.14)$ & 0.328 \\
\hline Dexamethasone (6 mg/day) started pre-ECMO & $74(47)$ & $16(18)$ & $58(82)$ & $<0.001$ \\
\hline Rescue therapy pre-ECMO & & & & \\
\hline
\end{tabular}


Table 1 (continued)

\begin{tabular}{|c|c|c|c|c|}
\hline \multirow[t]{2}{*}{ Characteristic } & \multirow[t]{2}{*}{ All $(N=159)$} & \multicolumn{2}{|l|}{ ICU admission } & \multirow[t]{2}{*}{$p$} \\
\hline & & Before July $1(N=88)$ & After July $1(N=71)$ & \\
\hline Any & $157(99)$ & $86(98)$ & $71(100)$ & 0.503 \\
\hline Neuromuscular blockade & $150(94)$ & $83(94)$ & $67(94)$ & 1.000 \\
\hline Prone positioning & $146(92)$ & $82(93)$ & $64(90)$ & 0.487 \\
\hline Inhaled nitric oxide or prostacyclin & $68(43)$ & $31(35)$ & $37(52)$ & 0.032 \\
\hline High-dose corticosteroids & $10(6)$ & $6(7)$ & $4(6)$ & 1.000 \\
\hline Almitrine & $1(0.6)$ & $1(1)$ & $0(0)$ & 1.000 \\
\hline Renal replacement therapy & $5(3)$ & $4(5)$ & $1(1)$ & 0.381 \\
\hline Cardiac arrest & $5(3)$ & $3(3)$ & $2(3)$ & 1.000 \\
\hline
\end{tabular}

Values are expressed as median (interquartile range) or number (\%)

ECMO extracorporeal membrane oxygenation, $I C U$ intensive care unit, MERT Mobile ECMO Retrieval Team, $\mathrm{PaO}_{2} / \mathrm{FiO}_{2}$ ratio of the partial pressure of arterial oxygen to the fraction of inspired oxygen, $P E E P$ positive end-expiratory pressure, $P B W$ predicted body weight, $P a C O_{2}$ partial pressure of arterial carbon dioxide, $R E S P$ Respiratory Extracorporeal Membrane Oxygenation Survival Prediction, $\mathrm{SaO}_{2}$ arterial oxygen saturation, SAPS Simplified Acute Physiology Score, SOFA Sequential Organ-Function Assessment

a Defined as chronic obstructive pulmonary disease or asthma

${ }^{b}$ Defined as hematological malignancies, active solid tumor or having received specific anti-tumor treatment within 1 year, solid-organ transplant or human immunodeficiency virus-infected, long-term corticosteroids or immunosuppressants

${ }^{c}$ Defined as plateau pressure minus PEEP

${ }^{\mathrm{d}}$ Mechanical power $(\mathrm{J} / \mathrm{min})=0.098 \times$ tidal volume $\times$ respiratory rate $\times$ peak pressure $-1 / 2 \times$ driving pressure. If not specified, peak pressure was considered equal to plateau pressure

intubation were significantly higher after July 1 , while the intubation-to-ECMO interval was comparable for the two periods. At ECMO cannulation, ventilation parameters, respiratory mechanics and blood gases did not differ between groups. More than $90 \%$ of the patients had received neuromuscular blocking agents and undergone prone-positioning before ECMO during both periods, while inhaled nitric oxide or prostacyclin $(35 \%$ vs. $52 \%$, $p=0.032)$ and dexamethasone $(18 \%$ vs. $82 \%, p<0.001)$ were used significantly more frequently after July 1 . Notably, D-dimer concentrations were significantly lower in patients admitted during the second period.

\section{On-ECMO patient management}

ECMO management during the two periods is described in Table 2 and Additional file 3. During the second period, more patients received airway pressure-release ventilation/bilevel-mode ventilation and two remained nonintubated, awake and on HFNO. Ventilation parameters were comparable during the two periods, with major decreases of driving pressure, static compliance, mechanical power and ventilatory ratio on ECMO-day 1. More than $80 \%$ of the patients were prone-positioned during ECMO, with comparable numbers of sessions during both periods, while a significantly lower percentage of patients received continuous neuromuscular blockade during the second period ( $94 \%$ vs. $27 \%, p<0.001)$. After July 1, 60 (85\%) patients received dexamethasone during the first 15 days on-ECMO and a significantly higher percentage of patients ( $15 \%$ vs. $37 \%, p=0.001)$ received high-dose corticosteroids for non-resolving ARDS [21].

\section{ICU and ECMO-related complications}

Despite significantly lower aPTT ratios during the first days on-ECMO after July 1, massive hemorrhage, hemorrhagic stroke and pulmonary embolism rates did not differ between periods. However, intravascular hemolysis and clogged circuits were more frequent after July 1 . Pneumothorax occurred significantly more frequently during the second period ( $6 \%$ vs. $17 \%, p=0.03$ ). The frequencies of antibiotic-treated ventilator-associated pneumonia and bacteremia episodes remained very high and comparable between study periods. More than a third of our patients required renal replacement therapy while on-ECMO.

\section{Patient outcomes}

On April 28, 2021, complete 90-day follow-up was obtained for all patients. The estimated state-occupation probabilities $(95 \% \mathrm{CI})$ of being on-ECMO, inICU \& weaned-off ECMO, alive and out of ICU or dead 90 days post-ECMO initiation, respectively, were: $1 \%(0.2-8 \%), 3 \%(1-10 \%), 59 \%(49-69 \%)$ and $36 \%$ (27-47\%) during the first period, and $3 \%(0.7-11 \%)$, $1 \%(0.2-10 \%), 48 \%(37-60 \%)$ and $48 \%(37-60 \%)$ for patients admitted after July 1 (Fig. 1 and Additional file 4). Kaplan-Meier estimates of 90-day survival were $64 \%$ and $52 \%$, respectively, for the first and the second periods (log-rank test $p=0.108$ ) (Fig. 2). After 
Table 2 Characteristics and complications during ECMO according to ICU admission before or after July 1, 2020

\begin{tabular}{|c|c|c|c|c|}
\hline \multirow[t]{2}{*}{ Characteristic } & \multirow[t]{2}{*}{ All $(N=159)$} & \multicolumn{2}{|l|}{ ICU admission } & \multirow[t]{2}{*}{$p$} \\
\hline & & Before July $1(n=88)$ & After July $1(n=71)$ & \\
\hline \multicolumn{5}{|l|}{ ECMO-day 1} \\
\hline Type of ECMO support & & & & 0.846 \\
\hline Femoral-jugular WV & $150(94)$ & $81(92)$ & $69(97)$ & \\
\hline Femoral-femoral VV & $4(3)$ & $3(3)$ & $1(1)$ & \\
\hline Ventilation mode & & & & $<0.001$ \\
\hline APRV/bilevel & $144(91)$ & $75(85)$ & $69(97)$ & \\
\hline Volume-assist control ventilation & $13(8)$ & $13(15)$ & $0(0)$ & \\
\hline High-flow oxygen & $2(1)$ & $0(0)$ & $2(3)$ & \\
\hline \multicolumn{5}{|l|}{ Ventilation parameters } \\
\hline Minute ventilation, $\mathrm{L} / \mathrm{min}$ & $3.8(2.4-6.2)$ & $3.5(2.4-6.1)$ & $4.6(2.6-6.2)$ & 0.455 \\
\hline Driving pressure, $\mathrm{cmH}_{2} \mathrm{O}$ & $12(12-14)$ & $12(12-14)$ & $12(12-14)$ & 0.623 \\
\hline Compliance, $\mathrm{mL} / \mathrm{cmH}_{2} \mathrm{O}$ & $13.5(9.5-22.1)$ & $13.2(9.0-20.1)$ & $13.9(10.0-23.3)$ & 0.120 \\
\hline Mechanical power, J/min & $7.2(4.3-12.1)$ & $6.4(4.1-11.3)$ & $8.1(5.1-12.3)$ & 0.220 \\
\hline Ventilatory ratio & $0.76(0.51-1.14)$ & $0.72(0.44-1.09)$ & $0.77(0.56-1.17)$ & 0.183 \\
\hline \multicolumn{5}{|l|}{ Laboratory values } \\
\hline Platelet, $\times 10^{3} / \mathrm{mm}^{3}$ & $247(177-317)$ & $237(177-310)$ & $266(179-333)$ & 0.320 \\
\hline Hemoglobin, g/dL & $9.4(8.1-10.7)$ & $9.1(8.0-10.3)$ & $10.0(8.5-11.0)$ & 0.023 \\
\hline Fibrinogen, mg/L & $7.1(5.7-8.2)$ & $6.7(5.7-8.1)$ & $7.1(5.6-8.2)$ & 0.711 \\
\hline D-Dimers, ng/L & $4905(2020-17,340)$ & $5935(2320-18,710)$ & $2485(1727-3655)$ & 0.020 \\
\hline aPTT ratio & $1.5(1.2-1.7)$ & $1.35(1.16-1.6)$ & $1.4(1.2-1.6)$ & 0.011 \\
\hline \multicolumn{5}{|l|}{ During the ECMO run } \\
\hline aPTT ratio ECMO-day 2 & $1.5(1.2-1.7)$ & $1.5(1.3-1.9)$ & $1.4(1.2-1.6)$ & 0.011 \\
\hline aPTT ratio ECMO-day 3 & $1.7(1.3-2.2)$ & $1.8(1.4-2.5)$ & $1.5(1.2-2.0)$ & 0.012 \\
\hline \multicolumn{5}{|l|}{ Adjuvant therapies on ECMO } \\
\hline Continuous neuromuscular blockers & $102(64)$ & $83(94)$ & $19(27)$ & $<0.001$ \\
\hline Prone positioning & $131(82)$ & $71(81)$ & $60(85)$ & 0.529 \\
\hline No. of sessions on-ECMO & $2(1-3)$ & $2(1-3)$ & $2(1-4)$ & 0.121 \\
\hline Nitric oxide or prostacyclin & $5(3)$ & $5(6)$ & $0(0)$ & 0.066 \\
\hline High-dose corticosteroids & $39(25)$ & $13(15)$ & $26(37)$ & 0.001 \\
\hline Renal replacement therapy & $63(40)$ & $39(44)$ & $24(34)$ & 0.178 \\
\hline Tracheostomy & $47(30)$ & $26(30)$ & $21(30)$ & 0.996 \\
\hline \multicolumn{5}{|l|}{ Received COVID-19 specific treatment } \\
\hline Remdesivir & $17(11)$ & $8(9)$ & $9(13)$ & 0.467 \\
\hline Lopinavir/ritonavir & $20(13)$ & $20(23)$ & $0(0)$ & $<0.001$ \\
\hline Tocilizumab & $9(6)$ & $8(9)$ & $1(1)$ & 0.042 \\
\hline Dexamethasone, $\leq 6 \mathrm{mg} /$ day $1^{\text {st }} 15$ days & $73(46)$ & $13(15)$ & $60(85)$ & $<0.001$ \\
\hline \multicolumn{5}{|l|}{ ECMO-related complications } \\
\hline Intravascular hemolysis & $29(18)$ & $9(10)$ & $20(28)$ & 0.004 \\
\hline Clogged circuit requiring change & $17(11)$ & $3(3)$ & $14(20)$ & $<0.001$ \\
\hline Severe thrombocytopenia $(<50 \mathrm{G} / \mathrm{L})^{\text {a }}$ & $10(6)$ & $5(6)$ & $5(7)$ & 0.754 \\
\hline ECMO setting/insertion change(s) ${ }^{b}$ & $8(5)$ & $4(5)$ & $4(6)$ & 0.051 \\
\hline Massive hemorrhage & $70(44)$ & $34(39)$ & $36(51)$ & 0.127 \\
\hline Stroke & & & & 1.000 \\
\hline Ischemic & $2(1)$ & $1(1)$ & $1(1)$ & \\
\hline Hemorrhagic & $8(5)$ & $4(5)$ & $4(6)$ & \\
\hline Pulmonary embolism & $23(14)$ & $16(18)$ & $7(10)$ & 0.138 \\
\hline Cardiac arrest & $17(11)$ & $9(10)$ & $8(11)$ & 0.833 \\
\hline Pneumothorax & $17(11)$ & $5(6)$ & $12(17)$ & 0.03 \\
\hline
\end{tabular}


Table 2 (continued)

\begin{tabular}{|c|c|c|c|c|}
\hline \multirow[t]{2}{*}{ Characteristic } & \multirow[t]{2}{*}{ All $(N=159)$} & \multicolumn{2}{|l|}{ ICU admission } & \multirow[t]{2}{*}{$p$} \\
\hline & & Before July $1(n=88)$ & After July $1(n=71)$ & \\
\hline Antibiotic-treated VAP & $159(100)$ & $75(85)$ & $63(89)$ & 0.516 \\
\hline$\geq 1$ treated bacteremia episode(s) & $75(47)$ & $44(51)$ & $31(44)$ & 0.388 \\
\hline
\end{tabular}

Values are expressed as median (interquartile range) or number (\%)

$A P R V$ airway pressure release ventilation, aPTT activated partial thromboplastin time, ECMO extracorporeal membrane oxygenation, ICU intensive care unit, $F r$ French, $\mathrm{FiO}_{2}$ the fraction of inspired oxygen, $P E E P$ positive end-expiratory pressure, $\mathrm{PaO}_{2}$ partial pressure of arterial oxygen, $\mathrm{PaCO}_{2}$ partial pressure of arterial carbon dioxide, PAPV positive airway-pressure ventilation, PBW predicted body weight, and SOFA Sequential Organ-Function Assessment, VA venoarterial, VV venovenous, VAP ventilator-associated pneumonia

${ }^{a}$ During the first 3 days

${ }^{\mathrm{b}}$ Included ECMO-cannulation switches from VA to VV; VA to venous-arteriovenous (V-AV); and VV to V-AV

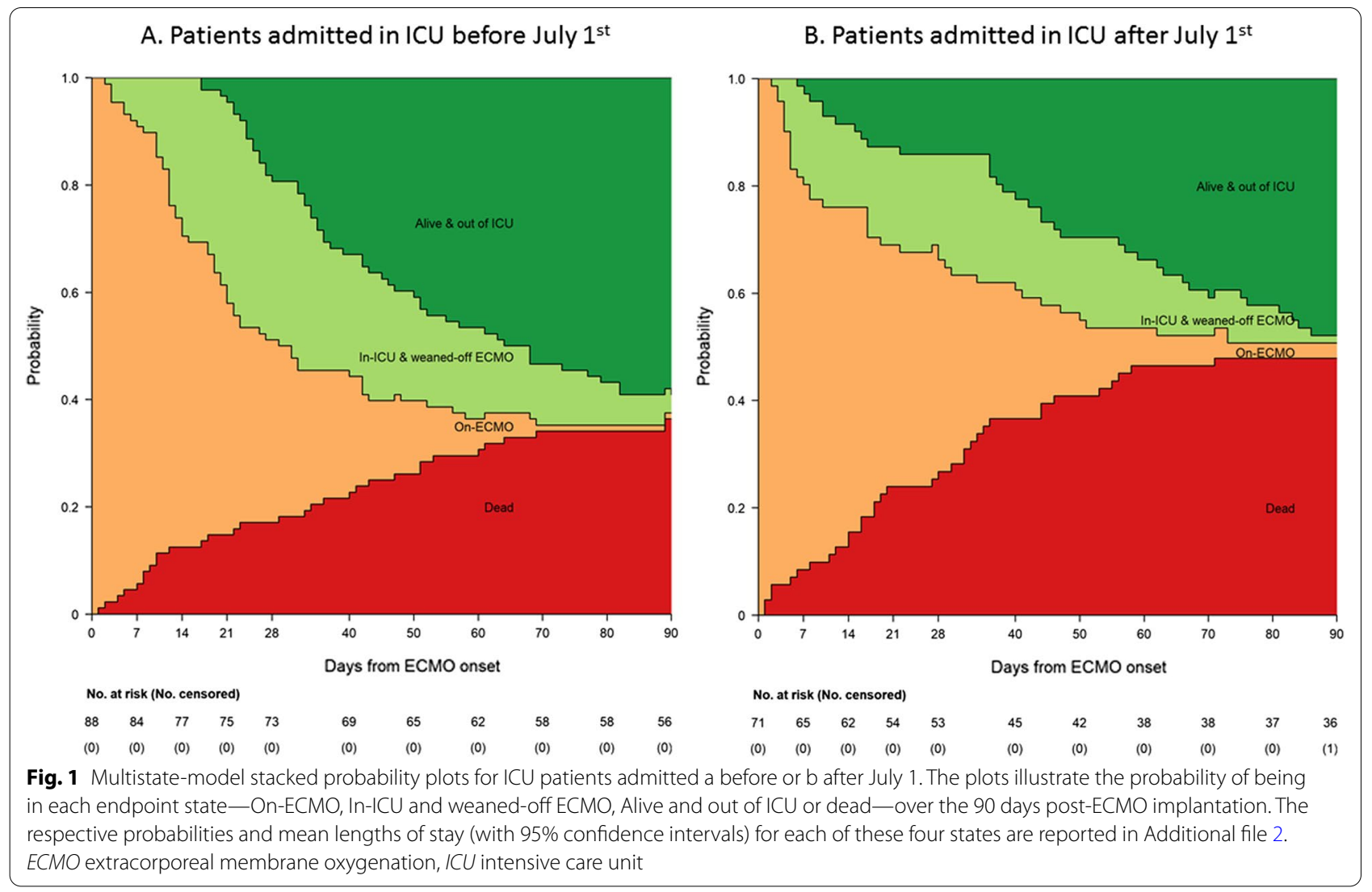

adjusting for confounders, patients treated after July 1 had a significantly higher probability of death by day 90 (HR 2.27, 95\% CI 1.02-5.07).

Median (IQR) ECMO durations during the first and second periods, respectively, were $19(10-35)$ and 18 $(5-35)$ days $(p=0.949)$, and $22(12-48)$ and $33(8-62)$ days among 90 -day survivors $(p=0.466)$. Similarly, ICU and hospital lengths of stay did not differ between periods (Additional file 5).

\section{Predictors of 90-day mortality}

The multivariable Cox regression model identified the following patient characteristics as being significantly associated with higher 90-day mortality: being admitted to the ICU after July 1 , older age and pre-ECMO SOFA cardiovascular component score $\geq 3$ (Table 3 ). In addition, a higher pre-ECMO $\mathrm{PCO}_{2}$ and longer ICU-admission-toECMO interval tended to be associated with higher 90-day mortality. Conversely, having received dexamethasone 


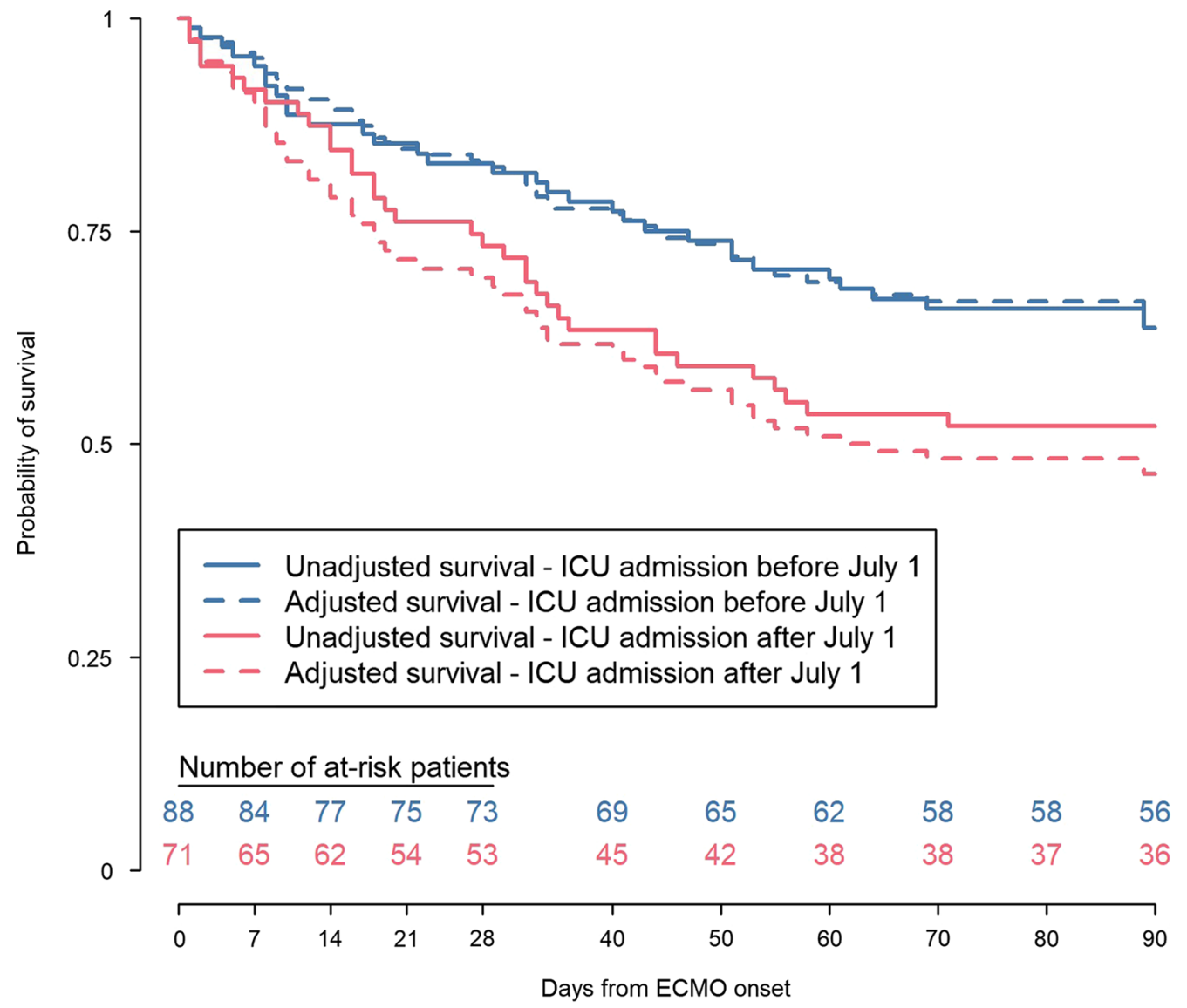

Fig. 2 90-day Kaplan-Meier survival estimates post-ICU admission according to admission period. Adjusted (HR 2.27, 95\% Cl 1.02-5.07; $p=0.05$ ) and unadjusted (log-rank test $p=0.108$ ) survival-model values

before ECMO implantation was a protective factor, whereas the SOFA renal component score, pre-ECMO driving pressure and $\mathrm{PaO}_{2} / \mathrm{FiO}_{2}$ ratio were not independently associated with higher risk of mortality. Multiple imputations of missing data yielded similar results but ICU admission after July 1 and dexamethasone treatment no longer reached statistical significance (Additional file 6).

\section{Discussion}

Patient mortality 90 days after starting ECMO support in our experienced center for the most severe forms of COVID-19 ARDS increased from 36\% before to $48 \%$ after July 1, 2020. Patients admitted after that date were older, had longer ICU-admission-to-intubation intervals, with more frequent HFNO or NIV use, and most of them had received dexamethasone at ECMO onset. Independent pre-ECMO predictors of 90-day mortality for the entire cohort were older age, longer ICU-admission-to-ECMO interval, cardiovascular dysfunction, not having received dexamethasone and being admitted after July 1, 2020.
The higher mortality rate of our ECMO-treated COVID-19 patients admitted after July 1, 2020, was unexpected. Differences in patient characteristics, management and SARS-CoV-2 pathogenicity might explain that observation. First, the responsibility of pathogenicity seems unlikely, since the original European SARSCoV-2 represented $>90 \%$ of strains circulating in France until mi-January 2021, after which the B.1.1.7 (Alpha) variant progressively became dominant. Second, most patient characteristics were comparable for the two periods, except for a 5-year higher median age during the second period. However, pre-ECMO patient management differed markedly after July 1, 2020. After RECOVERY trial results (published in June 2020) demonstrated lower mortality of patients randomized to receive 10 days of dexamethasone (6 mg/day) compared to usual care of mechanically ventilated COVID-19 patients (29\% vs. $41 \%$, respectively), most patients received corticosteroids. Pertinently, having started dexamethasone preECMO was significantly associated with lower 90-day 
Table 3 Factors at ECMO onset associated with 90-day mortality of severe COVID-19-ARDS patients on ECMO

\begin{tabular}{|c|c|c|c|c|}
\hline Factor & Univariate HR (95\% Cl) & $p$ & Multivariate $\mathrm{HR}(95 \% \mathrm{Cl})^{\text {a }}$ & $p$ \\
\hline Date of ICU admission & & 0.110 & & 0.050 \\
\hline Before July 1 & - & & - & \\
\hline After July 1 & $1.47(0.92-2.37)$ & & $2.27(1.02-5.07)$ & \\
\hline Age, years & & $<0.001$ & & 0.006 \\
\hline$<45$ & - & & - & \\
\hline $45-55$ & $2.81(1.26-6.29)$ & & $1.86(0.74-4.65)$ & \\
\hline$>55$ & $5.13(2.39-11.00)$ & & $3.55(1.48-8.50)$ & \\
\hline Days from ICU admission to ECMO & & 0.017 & & 0.066 \\
\hline$<4$ days & - & & - & \\
\hline 4-7 days & $1.37(0.69-2.74)$ & & $2.51(1.04-6.10)$ & \\
\hline $8-10$ days & $1.50(0.68-3.29)$ & & $2.83(1.04-7.70)$ & \\
\hline$>10$ days & $2.82(1.43-5.57)$ & & $3.02(1.15-7.92)$ & \\
\hline Driving pressure pre-ECMO, $\mathrm{cmH}_{2} \mathrm{O}$ & & 0.150 & & 0.264 \\
\hline$<17$ & - & & - & \\
\hline $17-20$ & $0.95(0.45-1.99)$ & & $0.84(0.37-1.94)$ & \\
\hline $20-22$ & $1.18(0.57-2.42)$ & & $1.04(0.47-2.29)$ & \\
\hline >22 & $1.98(1.03-3.81)$ & & $1.85(0.86-3.98)$ & \\
\hline $\mathrm{PaO}_{2} / \mathrm{FiO}_{2}$ pre-ECMO & & 0.621 & & 0.815 \\
\hline$<53$ & - & & - & \\
\hline $53-61$ & $0.80(0.42-1.53)$ & & $1.08(0.49-2.39)$ & \\
\hline $61-69$ & $0.82(0.42-1.61)$ & & $0.77(0.33-1.81)$ & \\
\hline '69 & $1.19(0.62-2.27)$ & & $1.12(0.48-2.62)$ & \\
\hline $\mathrm{PaCO}_{2}$ pre-ECMO, mmHg & & 0.007 & & 0.066 \\
\hline$<0$ & - & & - & \\
\hline $50-57$ & $0.72(0.31-1.67)$ & & $0.79(0.30-2.13)$ & \\
\hline $57-66$ & $1.64(0.82-3.30)$ & & $2.10(0.91-4.86)$ & \\
\hline '66 & $2.35(1.20-4.59)$ & & $2.11(0.98-4.53)$ & \\
\hline Dexamethasone started pre-ECMO & $1.07(0.67-1.71)$ & 0.783 & $0.37(0.17-0.79)$ & 0.012 \\
\hline SOFA score CV component $\geq 3$ & $1.82(1.13-2.94)$ & 0.013 & $2.44(1.31-4.55)$ & 0.004 \\
\hline SOFA score renal component $\geq 3$ & $1.58(0.87-2.89)$ & 0.156 & $1.50(0.72-3.13)$ & 0.296 \\
\hline
\end{tabular}

$\mathrm{CV}$ cardiovascular, SOFA sequential organ failure assessment, ICU intensive care unit, $H R$ hazard ratio, $C l$ confidence interval

${ }^{\text {a } C o m p l e t e ~ a n a l y s i s ~ o f ~} 55$ patients

mortality in our predictive model. Also, more frequent use of dexamethasone and high-dose corticosteroids for persistent ARDS after July 1 was not associated with a higher rate of infectious complications.

The other notable patient-management differences were more frequent HFNO or NIV use and for longer durations before intubation during the second period. Although noninvasive respiratory support was shown to lower the need for intubation and invasive mechanical ventilation, COVID-19 patients for whom this strategy failed may have been at increased risk of morbidity and mortality $[24,25]$. Indeed, strong and dysregulated spontaneous respiratory efforts, associated with wide transpulmonary pressure swings might heighten the risk of harmful "self-inflicted lung injury" on HFNO or
NIV, with more frequent fibrotic evolution of COVID19 pneumonia [26, 27]. That phenomenon could even be more difficult to detect in COVID-19 patients, who frequently experience dissociation between profound hypoxemic respiratory failure, and only moderate feelings and signs of respiratory distress and dyspnea [28]. Pertinently, the higher pneumothorax rate after July 1 , despite similar ventilatory management during both periods, supports that hypothesis, even though preECMO respiratory mechanic parameters did not differ significantly between periods. It should also be noted that although on-ECMO prone-positioning remained frequent (>80\%) after July 1, fewer patients received continuous neuromuscular blockade. Lastly, pulmonary embolism, stroke and hemorrhage rates did not 
change, while intravascular hemolysis and clogged circuits requiring change were more frequent after July 1. Those observations might reflect the significantly lower anticoagulation delivered to our second-period patients.

We acknowledge several limitations of this study. Our patients were treated in a high-volume, experienced ECMO center. Because better post-ECMO outcomes have been reported in such centers [4, 29], caution is required when extrapolating these results to less-experienced ECMO centers. Second, our study took place before SARS-CoV-2 variants associated with more severe ARDS forms became more prevalent (UK 20I/501Y.V1, South African 20H/501Y.V2, BR-P1 Brazilian, and Delta variants). ECMO outcomes of patients infected with those more virulent strains should be evaluated urgently. Third, COVID-19 management evolved throughout the study period, with widespread use of corticosteroids and interleukin-6 inhibitors. Therefore, patients refractory to several COVID-19 treatments who received ECMO during the second period might be considered sicker than those managed before July 1, 2020. Moreover, we cannot exclude that some residual confounding factors may not have been taken into account in our logistic model. Lastly, our cohort's mortality rate could evolve further, as some patients were still hospitalized 90 days after ICU admission.

\section{Conclusion}

In conclusion, survival of ECMO-rescued patients with severe COVID-19 ARDS in our experienced center has declined over time and is no longer comparable to that of non-COVID ECMO patients. Failure of prolonged noninvasive oxygenation strategies before intubation and increased lung damage, as well as selection of patients already refractory to specific COVID-19 treatments might partially explain this outcome. Although other residual confounding factors may not have been considered herein, the duration of noninvasive respiratory support, e.g., continuous positive airway pressure, HFNO or NIV, should perhaps be integrated in the decision to initiate ECMO for severe COVID-19 patients.

\footnotetext{
Abbreviations

aPTT: Activated partial thromboplastin time; ARDS: Acute respiratory distress syndrome; ECMO: Extracorporeal membrane oxygenation; VANV-ECMO: Venoarterial/venovenous-extracorporeal membrane oxygenation; ICU: Intensive care unit; COVID-19: Coronavirus disease 2019; HFNO: High-flow oxygen through a nasal cannula; NIV: Noninvasive ventilation; SAPS: Simplified Acute Physiology Score; SARS-CoV-2: Severe acute respiratory syndrome coronavirus-2; RESP: Respiratory extracorporeal membrane oxygenation survival prediction; SOFA: Sequential organ-failure assessment.
}

\section{Supplementary Information}

The online version contains supplementary material available at https://doi. org/10.1186/s13054-021-03780-6.

Additional file 1. All possible transition probabilities from one state to another over time.

Additional file 2. The estimated probabilities ( $95 \% \mathrm{Cl}$ ) of all possible transitions from one state to another for (A) 88 patients admitted to the ICU before July 1, 2020, and (B) 71 patients admitted to the ICU after July 1, 2020.

Additional file 3. Additional information concerning patients' characteristics and complications on-ECMO according to ICU-admission period.

Additional file 4. Probabilities of being in each of the four endpoint states and the mean time spent in each one on days $28,40,50,60$, and 90 postECMO onset according to the ICU-admission date.

Additional file 5. ECMO duration, and ICU and hospital lengths of stay according to the ICU-admission period.

Additional file 6. Predictive factors associated with 90-day mortality of 159 adults with COVID-19 severe ARDS with multiple imputations.

\section{Acknowledgements}

GRC-RESPIRE Sorbonne Université:

Groupe de Recherche Clinique en REanimation et Soins intensifs du Patient en Insuffisance Respiratoire aiguE.

\section{Authors' contributions}

$M S$, EL, SAJ, JC, NB, PB, LL, MP, GE, CEL, MD, JMC, MF, PL, GL, and AC were involved in data generation. MS, $\mathrm{DH}$, and $\mathrm{AC}$ were involved in the analysis of the data. MS, DH, and AC wrote the manuscript. All authors contributed to critical reading of the text and its revision. All authors read and approved the final manuscript.

\section{Funding}

No funding was received for this study. The corresponding author had full access to all data and the final responsibility to submit for publication.

\section{Availability of data and materials}

The datasets used during the current study are available from the corresponding author on reasonable request.

\section{Declarations}

\section{Ethics approval and consent to participate}

The Sorbonne University Ethics Committee (CER-SU-2020-46) approved the study protocol. Moreover, the dataset used for the analysis was anonymized.

\section{Consent for publication}

Not applicable.

\section{Competing interests}

Matthieu Schmidt reports lecture fees from Getinge, Drager, and Xenios outside the submitted work. Martin Dres reports personal fees from Lungpacer, outside the submitted work. Muriel Fartoukh reports non-financial support from bioMérieux, and personal fees from Pfizer, bioMérieux, Fisher \& Paykel, and grants from French Ministry of Health, outside the submitted work. Jean-Michel Constantin reports personal fees and non-financial support from Drager, GE Healthcare, Sedana Medical, Baxter, and Amomed; personal fees from Fisher, Paykel Healthcare, Orion, Philips Medical, and Fresenius Medical Care; and non-financial support from LFB, and Bird Corporation, outside of the submitted work. Alain Combes reports grants from Getinge, personal fees from Getinge, Baxter, and Xenios outside the submitted work. The other authors declare that they have no conflicts of interest related to this manuscript. 


\begin{abstract}
Author details
IInserm, UMRS_1166-ICAN, Institute of Cardiometabolism and Nutrition, Sorbonne Université, Paris, France. ${ }^{2}$ Service de Médecine Intensive-Réanimation, iCAN, Institut de Cardiologie, Assistance Publique-Hôpitaux de Paris (APHP), Sorbonne Université Hôpital Pitié-Salpêtrière, 47, Bd de L'Hôpital, 75651 Paris Cedex 13, France. ${ }^{3}$ GRC 30, RESPIRE, APHP, Hôpital Pitié-Salpêtrière, Sorbonne Université, Paris, France. ${ }^{4}$ INSERM, Institut Pierre-Louis d'Epidémiologie Et de Santé Publique, APHP, Hôpitaux Universitaires Pitié-Salpêtrière Charles Foix, Département de Santé Publique, Centre de Pharmacoépidémiologie (Cephepi), Sorbonne Université, CIC-1421 Paris, France. ${ }^{5}$ APHP, Hôpital Pitié-Salpêtrière, Service de Pneumologie, Médecine Intensive-Réanimation (Département "R3S"), Sorbonne Université, Paris, France. ${ }^{6}$ Inserm, UMRS_1158 Neurophysiologie Respiratoire Expérimentale Et Clinique, Sorbonne Université, Paris, France. ${ }^{7}$ GRC 29, APHP, DMU DREAM, Department of Anaesthesiology and Critical Care, Pitié-Salpêtrière Hospital, Sorbonne University, Paris, France. ${ }^{8}$ APHP, Sorbonne Université, Hôpital Tenon, Service de Médecine Intensive Réanimation, Sorbonne Université, Paris, France. ${ }^{9}$ Service de Chirurgie Cardiaque, Institut de Cardiologie, APHP, Paris, Sorbonne, France.
\end{abstract}

Received: 28 July 2021 Accepted: 30 September 2021

Published online: 09 October 2021

\section{References}

1. Barbaro RP, MacLaren G, Boonstra PS, Iwashyna TJ, Slutsky AS, Fan E, et al. Extracorporeal membrane oxygenation support in COVID-19: an international cohort study of the Extracorporeal Life Support Organization registry. Lancet. 2020;396:1071-8.

2. Schmidt M, Hajage D, Lebreton G, Monsel A, Voiriot G, Levy D, et al. Extracorporeal membrane oxygenation for severe acute respiratory distress syndrome associated with COVID-19: a retrospective cohort study. Lancet Respir Med. 2020;8:1121-31.

3. Diaz RA, Graf J, Zambrano JM, Ruiz C, Espinoza JA, Bravo Sl, et al. ECMO for COVID-19-associated severe ARDS in Chile: a nationwide incidence and cohort study. Am J Respir Crit Care Med. 2021;204:34-43.

4. Lebreton G, Schmidt M, Ponnaiah M, Folliguet T, Para M, Guihaire J, et al. Extracorporeal membrane oxygenation network organisation and clinical outcomes during the COVID-19 pandemic in Greater Paris, France: a multicentre cohort study. Lancet Respir Med. 2021;9:851-62.

5. Combes A, Hajage D, Capellier G, Demoule A, Lavoué S, Guervilly C, et al. Extracorporeal membrane oxygenation for severe acute respiratory distress syndrome. N Engl J Med. 2018;378:1965-75.

6. Schmidt M, Pham T, Arcadipane A, Agerstrand C, Ohshimo S, Pellegrino $\checkmark$, et al. Mechanical ventilation management during extracorporeal membrane oxygenation for acute respiratory distress syndrome. An international multicenter prospective cohort. Am J Respir Crit Care Med. 2019;200:1002-12.

7. Shaefi S, Brenner SK, Gupta S, O'Gara BP, Krajewski ML, Charytan DM, et al. Extracorporeal membrane oxygenation in patients with severe respiratory failure from COVID-19. Intensive Care Med. 2021;47:208-21.

8. RECOVERY Collaborative Group, Horby P, Lim WS, Emberson JR, Mafham $\mathrm{M}$, Bell $\mathrm{J}$, et al. Dexamethasone in hospitalized patients with Covid-19preliminary report. N Engl J Med. 2021;384(8):693-704.

9. Le Gall JR, Lemeshow S, Saulnier F. A new Simplified Acute Physiology Score (SAPS II) based on a European/North American multicenter study. JAMA. 1993;270:2957-63.

10. Bréchot N, Mastroianni C, Schmidt M, Santi F, Lebreton G, Hoareau A-M, et al. Retrieval of severe acute respiratory failure patients on extracorporeal membrane oxygenation: any impact on their outcomes? J Thorac Cardiovasc Surg. 2018;155:1621-9.e2.

11. Beurtheret S, Mordant P, Paoletti X, Marijon E, Celermajer DS, Léger P, et al. Emergency circulatory support in refractory cardiogenic shock patients in remote institutions: a pilot study (the cardiac-RESCUE program). Eur Heart J. 2013;34:112-20.

12. Hékimian $\mathrm{G}$, Lebreton $\mathrm{G}$, Bréchot $\mathrm{N}$, Luyt C-E, Schmidt M, Combes A. Severe pulmonary embolism in COVID-19 patients: a call for increased awareness. Crit Care. 2020;24:274.
13. Guervilly C, Prud'homme E, Pauly V, Bourenne J, Hraiech S, Daviet F, et al. Prone positioning and extracorporeal membrane oxygenation for severe acute respiratory distress syndrome: time for a randomized trial? Intensive Care Med. 2019;45:1040-2.

14. COVID-ICU Group on behalf of the REVA Network and the COVID-ICU Investigators. Clinical characteristics and day-90 outcomes of 4244 critically ill adults with COVID-19: a prospective cohort study. Intensive Care Med. 2021;47:60-73.

15. Combes A, Schmidt M, Hodgson CL, Fan E, Ferguson ND, Fraser JF, et al. Extracorporeal life support for adults with acute respiratory distress syndrome. Intensive Care Med. 2020;46:2464-76.

16. Schmidt M, Fisser C, Martucci G, Abrams D, Frapard T, Popugaev K, et al. Tracheostomy management in patients with severe acute respiratory distress syndrome receiving extracorporeal membrane oxygenation: an international multicenter retrospective study. Crit Care. 2021;25:238.

17. Vincent JL, Moreno R, Takala J, Willatts S, De Mendonça A, Bruining $H$, et al. The SOFA (Sepsis-related Organ Failure Assessment) score to describe organ dysfunction/failure. On behalf of the Working Group on Sepsis-Related Problems of the European Society of Intensive Care Medicine. Intensive Care Med. 1996;22:707-10.

18. Schmidt M, Bailey M, Sheldrake J, Hodgson C, Aubron C, Rycus PT, et al. Predicting survival after extracorporeal membrane oxygenation for severe acute respiratory failure. The Respiratory Extracorporeal Membrane Oxygenation Survival Prediction (RESP) score. Am J Respir Crit Care Med. 2014;189:1374-82.

19. Gattinoni L, Tonetti T, Cressoni M, Cadringher P, Herrmann P, Moerer O, et al. Ventilator-related causes of lung injury: the mechanical power. Intensive Care Med. 2016;42:1567-75.

20. Sinha P, Calfee CS, Beitler JR, Soni N, Ho K, Matthay MA, et al. Physiological analysis and clinical performance of the ventilatory ratio in acute respiratory distress syndrome. Am J Respir Crit Care Med. 2018;199:333-41.

21. Meduri GU, Headley AS, Golden E, Carson SJ, Umberger RA, Kelso T, et al. Effect of prolonged methylprednisolone therapy in unresolving acute respiratory distress syndrome: a randomized controlled trial. JAMA. 1998:280:159-65.

22. Putter H, Fiocco M, Geskus RB. Tutorial in biostatistics: competing risks and multi-state models. Stat Med. 2007;26:2389-430.

23. Sterne JAC, White IR, Carlin JB, Spratt M, Royston P, Kenward MG, et al. Multiple imputation for missing data in epidemiological and clinical research: potential and pitfalls. BMJ. 2009;338:b2393.

24. Valley TS, Walkey AJ, Lindenauer PK, Wiener RS, Cooke CR. Association between noninvasive ventilation and mortality among older patients with pneumonia. Crit Care Med. 2017;45:e246-54.

25. Kang BJ, Koh Y, Lim C-M, Huh JW, Baek S, Han M, et al. Failure of high-flow nasal cannula therapy may delay intubation and increase mortality. Intensive Care Med. 2015;41:623-32.

26. Brochard L, Slutsky A, Pesenti A. Mechanical ventilation to minimize progression of lung injury in acute respiratory failure. Am J Respir Crit Care Med. 2017;195:438-42.

27. Brochard L. Ventilation-induced lung injury exists in spontaneously breathing patients with acute respiratory failure: yes. Intensive Care Med. 2017;43:250-2.

28. Wilkerson RG, Adler JD, Shah NG, Brown R. Silent hypoxia: a harbinger of clinical deterioration in patients with COVID-19. Am J Emerg Med. 2020;38(2243):e5-6.

29. Barbaro RP, Odetola FO, Kidwell KM, Paden ML, Bartlett RH, Davis MM, et al. Association of hospital-level volume of extracorporeal membrane oxygenation cases and mortality. Analysis of the extracorporeal life support organization registry. Am J Respir Crit Care Med. 2015;191:894-901.

\section{Publisher's Note}

Springer Nature remains neutral with regard to jurisdictional claims in published maps and institutional affiliations. 